Int. J. Dev. Biol. 55: 447-453

doi: $10.1387 / \mathrm{ijdb} .103222 \mathrm{rn}$

\title{
Paracrine regulation of angiogenesis by different cell types in the aorta ring model
}

\author{
ROBERTO F. NICOSIA* ${ }^{* 1,2}$, PENELOPE ZORZI², GIOVANNI LIGRESTI ${ }^{2}$, ANN MORISHITA ${ }^{2}$ and ALFRED C. APLIN ${ }^{2}$ \\ ${ }^{1}$ Pathology and Laboratory Medicine Services, Veterans Administration Puget Sound Health Care System, Seattle, \\ WA and ${ }^{2}$ Department of Pathology, University of Washington, Seattle, WA, USA
}

\begin{abstract}
The development of blood vessels during angiogenesis is the result of paracrine interactions between tube-forming endothelial cells and angiogenic factor-producing nonendothelial cells. This process can be reproduced and studied under chemically defined culture conditions by culturing vascular explants in three-dimensional gels of extracellular matrix. Rings of rat or mouse aorta cultured in collagen, fibrin or basement membrane gels produce angiogenic outgrowths composed of a mixed population of endothelial cells and nonendothelial cells. Aortic angiogenesis is regulated by endogenous angiogenic factors, inflammatory cytokines, chemokines, extracellular matrix molecules, and proteolytic enzymes produced by cells of the vessel wall in response to the injury of the dissection procedure. In this paper, we review how macrophages, mural cells and fibroblasts regulate different stages of the angiogenic process, from the formation of immature endothelial sprouts to the reabsorption of the neovessels. We also describe how aortic cultures can be used to study interactions between angiogenic outgrowths and nonvascular cell types such as bone marrow macrophages, platelets or cancer cells. Morphologic, genetic and functional studies of this model have provided invaluable information on how vessels form, mature, interact with nonvascular cell types, and are eventually reabsorbed. Further analysis of the paracrine cross-talk between aortic endothelial and nonendothelial cells is likely to provide new insights into the angiogenic process and its key mechanisms.
\end{abstract}

KEY WORDS: VEGF, bFGF, PDGF, angiopoietin, MMP, TIMP, microarray

\section{Introduction}

Angiogenesis, the process of formation of blood vessels, contributes to the progression of many diseases including cancer and atherosclerosis (Carmeliet, 2003). New vessels develop by sprouting from pre-existing vessels, splitting of parent vessels into daughter vessels, or in situ differentiation from primitive mesenchymal cells and circulating endothelial progenitor cells (Patan, 2004).

Over the past four decades, many experimental models have been developed to study angiogenesis and its mechanisms (Ribatti and Vacca, 1999; Auerbach et al., 2003; Ucuzian and Greisler, 2007). Among these the ex vivo aortic ring model of angiogenesis combines advantages of in vivo and in vitro models. In this system rings of rat or mouse aorta embedded in gels of extracellular matrix generate new vessels in response to the injury of the dissection procedure (Nicosia and Ottinetti, 1990; Masson et al., 2002; Zhu et al., 2003b; Aplin et al., 2008). Aortic outgrowths are composed of a mixed population of endothelial cells, pericytes, fibroblasts, and macrophages. Angiogenesis in aortic cultures is mediated by paracrine interactions between cells of the vessel wall and can be modulated by incorporating into the system exogenous cells or by supplementing the culture medium with molecular regulators of angiogenesis (Nicosia, 2009). In this paper we describe how the aortic ring model can be used to study paracrine mechanisms of angiogenesis regulation and the role of different cell types in the induction, maturation, and resolution of the angiogenic process.

\section{Angiogenesis in the aortic ring model is induced by injury}

Rings of rat aorta embedded in gels of interstitial collagen and cultured in a growth medium optimized for endothelial cells produce

\footnotetext{
Abbreviations used in this paper: bFGF, basic fibroblast growth factor; DT, diphtheria toxin; IL, interleukin; MMP, matrix metalloproteinase; TIMP, tissue inhibitor of MMP; TNF, tumor necrosis factor; VEGF, vascular endothelial growth factor.
}

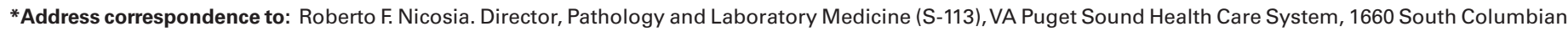
Way, Seattle, WA 98108, USA. Fax: +1-206-764-2001. e-mail: roberto.nicosia@va.gov

Final, author-corrected PDF published online: 27 July 2011.
} 
angiogenic outgrowths spontaneously and in the absence of serum or exogenous stimuli (Nicosia and Ottinetti, 1990). The angiogenic response of the aortic explants is a self limited process triggered by the injury of the dissection procedure. Endothelial sprouts emerge from the cut edges of the explants at day 2-3, propagate into the gel for several days, stop growing during the second week of culture, and are gradually reabsorbed.

Angiogenesis in aortic cultures is driven by growth factors, inflammatory cytokines and chemokines produced by the explants. Among these is basic fibroblast growth factor (bFGF), which is rapidly released from cell and extracellular matrix depots during the first day of culture (Villaschi and Nicosia, 1993). Vascular endothelial growth factor A (VEGF) is concurrently upregulated during the first 24 hours of culture (Fig. 1). The concentration of bFGF and VEGF in the aortic ring conditioned medium is highest during the first few days of culture and decreases to undetectable levels over time as vessels stop growing and regress (Villaschi and Nicosia, 1993; Nicosia et al., 1997). Additional factors upregulated during the first day of culture include placenta growth factor (PIGF), hepatocyte growth factor (HGF), transforming growth- $\beta 1$ (TGF- $\beta 1$ ), Kit ligand, platelet derived growth factor A (PDGFA), PDGFB, and VEGFB. Proangiogenic inflammatory cytokines include interleukin-6 (IL-6), growth differentiation factor15 (GDF15), macrophage inhibitory factor (MIF), and tumor necrosis factor- $\alpha$ (TNF)(Nilsson et al., 2005; Huh et al., 2010; Amin et al., 2003; Leibovich et al., 1987). Proangiogenic chemokines include CX3CL1 (Fraktalkine), CCL2 (MCP1), CXCL1 (Gro- $\alpha$ ), CXCL2 (Gro-9), CXCL3 (Gro- $\gamma$ ), and CXCL-12 (SDF-1) (Table 1) (Strieter et al., 2004). bFGF, VEGF (Fig. 2) and many of the growth factors, cytokines and chemokines upregulated in aortic ring cultures stimulate the angiogenic response of the aortic rings when added as exogenous molecules to the system (Gelati et al., 2008; Aplin et al., 2010; Salcedo et al., 1999; Nicosia et al., 1994a; Nicosia, 2009).

Anumber of observations underscore the importance of endogenous growth factors and inflammatory cytokines and chemokines as mediators of angiogenesis in the aortic ring model. (A) Blockade of bFGF and VEGF with neutralizing antibodies or signal transduction antagonists significantly decreases the angiogenic response of the explants (Villaschi and Nicosia, 1993; Nicosia et al., 1997; Emanuel et al., 2004). (B) Pharmacologic inhibition or genetic disruption of

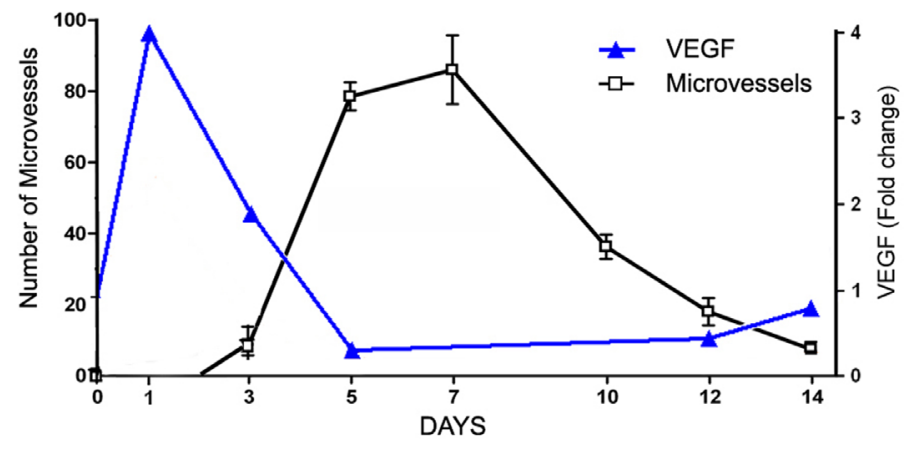

Fig. 1. Relationship between vascular endothelial growth factor (VEGF) expression and angiogenic response in collagen gel cultures of rat aorta. Quantitative real-time PCR shows that VEGF is rapidly upregulated prior to the sprouting of neovessels. Angiogenesis is followed by vascular regression during the second week of culture when VEGF expression is reduced to baseline levels.
CXCR2, the receptor of CXCL1, CXCL2, and CXCL3, reduces aortic angiogenesis (Gelati et al., 2008). (C) A cocktail of cytokines and chemokines upregulated in aortic ring cultures (TNF, IL1- $\alpha$, CXCL1, CCL3, CCL4,) synergistically stimulates the angiogenic response of aortic rings to a low dose of VEGF which is minimally stimulatory when used alone (Aplin et al., 2006). (D) Aortic rings lose their ability to spontaneously sprout if embedded in collagen when they are no longer releasing growth factors, i.e. 10-14 days after the initial dissection; these quiescent rings remain viable and can be stimulated to sprout by re-injury or with exogenous bFGF or VEGF (Nicosia et al., 1997; Aplin et al., 2008). (E) The intensity of the angiogenic response is strictly dependent on the volume in which the aortic rings are cultured, and weakens as the volume of the growth medium is expanded and the endogenous growth factors are diluted (Aplin et al., 2008) (Fig. 3). (F) Medium conditioned by aortic rings stimulates the angiogenic response of other rings (Villaschi and Nicosia, 1993). (G) Aortic rings stimulate the angiogenic response of other vessel explants including rings of vena cava in collagen gel co-cultures (Nicosia et al., 2005).

\section{Cellular composition of the aortic outgrowths}

Angiogenic sprouting in aortic cultures is preceded by migration of adventitial macrophages and fibroblasts into the periaortic gel matrix. Once vessel sprouts emerge from the explants, they generate networks by elongating, branching, and forming anastomotic loops through endothelial migration and proliferation. As they mature, neovessels become invested with pericytes which migrate and proliferate at the abluminal surface of the endothelium (Nicosia and Ottinetti, 1990; Nicosia and Villaschi, 1995) (Fig. 4). Macrophages accumulate primarily at the roots of the vascular outgrowths where they are closely associated with endothelial cells and pericytes (Gelati et al., 2008). Some macrophages move along the stalks of the neovessels or into the gel matrix adjacent to the vessel outgrowths. After the angiogenic growth phase, the aortic neovasculature is reabsorbed through fragmentation and retraction of the endothelial tubes. Vascular regression is associated with periaortic lysis of the gel and accumulation of macrophages in the area of lysis and at the base of the involuted outgrowth (Zhu et al., 2000).

\section{Origin of microvessels in aortic cultures}

Neovessels in aortic cultures arise primarily from the cut edges of the explants where endothelial cells and subendothelial mural cells of the intimal layer migrate over the wounded aortic wall into the surrounding collagen (Villaschi and Nicosia, 1993). Exposure of the luminal surface of the intimal endothelium to collagen forces endothelial cells to reorganize into capillary tubes. The capacity of intimal endothelial cells to switch to a microvascular phenotype can be directly demonstrated by culturing in collagen gels whole aortas turned inside out and ligated at both ends (Nicosia et al., 1992; Mori et al., 1988). This method exposes the luminal surface of the endothelium to collagen while blocking the outgrowth of adventitial cells. The intimal endothelial cells reorganize into microvessels and sprout into the collagen matrix. The angioformative properties of the intimal endothelium can be further demonstrated by embedding isolated aortic endothelial cells in collagen gel or by treating them with angiogenic factors (Nicosia etal., 1994b). In addition rat carotid explants fail to generate an angiogenic response when completely 

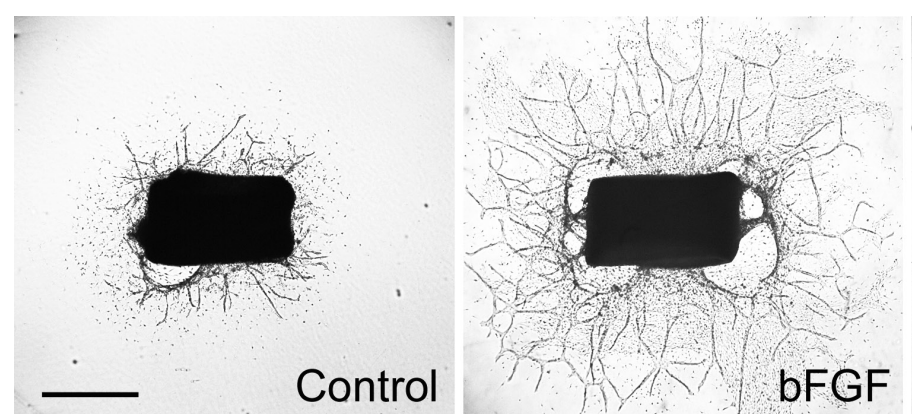

de-endothelialized with a balloon catheter whereas control carotid arteries with an intact intimal endothelium produce microvessels from their ends of resection (Nicosia, 2009). These findings indicate that the intimal layer of the aortic wall plays a central role in the sprouting of the aortic explants. However the angiogenic response of the aortic rings also requires an intact adventitia since selective killing of the adventitial layer with ethanol abrogates angiogenesis (Diglio et al., 1989). Although this procedure causes loss of adven-

\section{TABLE 1}

\section{GROWTH FACTORS, CYTOKINES AND CHEMOKINES UPREGULATED IN ONE-DAY-OLD SERUM-FREE COLLAGEN GEL CULTURES OF RAT AORTA}

\begin{tabular}{lllc} 
Accession number & Gene Symbol & Description & Fold Change* \\
\hline NM_001017496 & CXCL13 (BLC) & B-lymphocyte chemoattractant & 35.0 \\
NM_001105962 & PRG4 & Hemangiopoietin & 24.0 \\
NM_134455 & CX3CL1 & Fraktalkine & 15.0 \\
NM_031530 & CCL2 (MCP-1) & Monocyte chemotactic protein 1 & 14.9 \\
NM_012589 & IL6 & Interleukin 6 & 7.8 \\
NM_001007612 & CCL7 (MCP-3) & Monocyte chemotactic protein 3 & 6.8 \\
NM_013025 & CCL3 (MIP-1a) & Macrophage inflammatory protein 1 & 6.0 \\
NM_139089 & CXCL10 (IP-10) & Interferon u61543 induced protein 10 & 5.7 \\
NM_019216 & MIC1/GDF15 & Growth differentiation factor 15 & 4.7 \\
NM_053595 & PGF & Placental growth factor & 4.0 \\
NM_031051 & MIF & Macrophage inhibitory factor & 3.6 \\
NM_017017 & HGF & Hepatocyte growth factor & 3.5 \\
NM_053858 & CCL4 (MIP-1b) & Macrophage inflammatory protein 2 & 3.2 \\
NM_021578 & TGFb1 & Transforming growth factor beta 1 & 3.1 \\
NM_021843 & KitL & Kit ligand & 3.0 \\
NM_022182 & FGF7 & Fibroblast growth factor 7 & 2.9 \\
NW_001084807 & NGF & Nerve growth factor & 2.7 \\
NM_001033883 & CXCL12 (SDF-1a) & Stromal derived factor 1 & 2.7 \\
NM_053647 & CXCL2 & Growth related oncogene beta & 2.6 \\
NM_182952 & CXCL11 & I-TAC & 2.6 \\
NM_133519 & IL11 & Interleukin 11 & 2.6 \\
NM_013174 & TGFb3 & Transforming growth factor beta 3 & 2.2 \\
NM_001105822 & CCL12 (MCP-5) & Monocyte chemotactic protein 5 & 2.2 \\
NM_012801 & PDGFA & Platelet derived growth factor alpha & 2.1 \\
NM_030845 & CXCL1 (Gro-a) & Growth related oncogene alpha & 2.0 \\
NM_001017478 & CXCL16 & NCC-4 & 2.0 \\
NM_053549 & VEGFB & Vascular endothelial growth factor B & 1.8 \\
NM_031512 & 1L1b & Interleukin 1 beta & 1.7 \\
NM_001105749 & IL16 & Interleukin 16 & 1.5 \\
NM_031836 & VEGFA & Vascular endothelial growth factor A & 1.6 \\
NM_012675 & TNF & Tumor necrosis factor & 1.6 \\
NM_138522 & CXCL3 (GRO-g) & Growth related oncogene gamma & 1.5 \\
\hline Dat & CCL20 (MIP-3a) & Macrophage inflammatory protein 3 & 1.5 \\
\hline
\end{tabular}

Data shown in this table demonstrate fold increase in gene expression by microarray analysis at day 1 compared to time zero (freshly isolated aorta). Microarray analysis was performed on RNA samples labeled and hybridized to Affymetrix Rat Genome 230-2.0 gene chips. Fold-change in gene expression was calculated based on RMA values. Statistical significance was set at $p<0.05$.

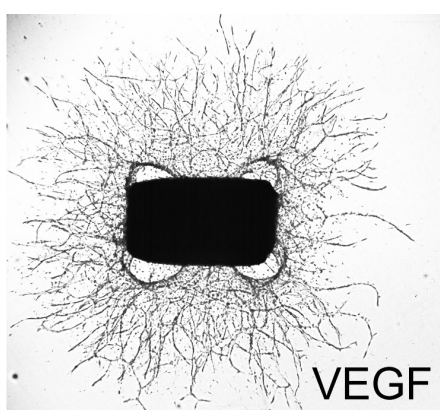

Fig. 2. Stimulatory effect of recombinant angiogenic factors in the aortic ring assay. Photomicrographs illustrate the angiogenic response in collagen gel cultures of an untreated aortic ring (control) and rings treated with basic fibroblast growth factor (bFGF, $25 \mathrm{ng} / \mathrm{ml}$ ) orvascular endothelial growth factor (VEGF, $10 \mathrm{ng} / \mathrm{ml}$ ). Scale bar, $1 \mathrm{~mm}$.

titial vasa vasorum, which if present contribute to the angiogenic response, recent evidence from our laboratory point to the resident innate immune system as the source of critical proangiogenic stimuli in the aortic ring model (Gelati et al., 2008).

\section{Role of adventitial macrophages as injury sensors and transducers of the angiogenic response}

Genetic analysis of aortic cultures demonstrates widespread activation of the aortic immune system prior to angiogenic sprouting. Upregulated genes include many macrophage-stimulatory cytokines and chemokines (Table 1). Immunohistochemical studies show that the angiogenic response is associated with mobilization from the aortic adventitia of CD45+ CD11b+ and CD68+ macrophages (Aplin et al., 2006). Ablation of macrophages in rat aortic cultures with liposomal clodronate, a toxic compound that selectively kills phagocytic cells, markedly impairs VEGF production and angiogenic sprouting. Similar results are obtained by treating with diphtheria toxin (DT) aortic cultures from transgenic mice carrying the human DT receptor under the control of the CD11b promoter (Gelati et al., 2008). DT in these cultures selectively ablates CD11b+ macrophages but not the other cell types which are not sensitive to the toxic effects of DT. The angiogenic response in macrophage-ablated aortic cultures is restored by adding exogenous VEGF. VEGF production and angiogenesis can also be reactivated by replenishing macrophage-depleted aortic cultures with bone marrow derived macrophages. This is accomplished by co-embedding in collagen gels bone marrow macrophages with rat aortic rings pre-treated

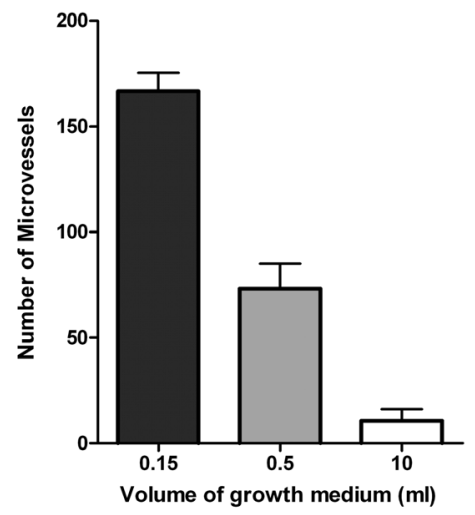

Fig. 3. The angiogenic response of aortic rings is inversely related to the volume of the culture medium. Peak of growth measurements of angiogenic outgrowths in aortic ring culture prepared in 96-well 10.15 $\mathrm{ml})$, 4-well $(0.5 \mathrm{ml})$, or $10 \mathrm{~cm}(10 \mathrm{ml})$ dishes demonstrate that dilution of endogenous growth factors obtained by increasing the volume of the culture medium impairs angiogenesis. 
with liposomal clodronate (Gelati et al., 2008). Close examination of these co-cultures shows a gradient of macrophage survival: macrophages close to the aortic rings appear healthy whereas macrophages away from the explants become granular and disintegrate over time. These experiments demonstrate the existence of mechanisms of reciprocal paracrine stimulation between cells of the vessel wall and macrophages.

\section{Role of macrophages as regulators of vascular regression}

Following the angiogenic response, aortic outgrowths involute and neovessels are gradually reabsorbed. The vascular regression process is associated with increased production and accumulation of matrix metalloproteinases including MMP2, MMP3, MMP9, MMP12, and MMP14 (Zhu et al., 2000; Aplin et al., 2009). Treatment of aortic outgrowths with synthetic MMP inhibitors blocks the lysis of the collagen matrix, stabilizes the neovessels and significantly delays the reabsorption of the neovasculature. Inhibition of vascular regression is also obtained with TIMP2, TIMP3 and TIMP4, all of which inhibit MMP14, but also with TIMP1 which is a poor inhibitor of this enzyme. Vascular regression can be delayed but not completely blocked with anti-MMP14 antibodies. Studies of aortic cultures from genetically modified mice with disrupted MMP2 and MMP9 show that these enzymes have no significantly effects on the angiogenic response of the aortic rings. Conversely TIMP2, TIMP3, TIMP4 and anti-MMP14 antibodies, but not TIMP1, inhibit angiogenesis when added to the aortic cultures from the beginning of the experiment (Aplin et al., 2009). These studies indicate that MMP14, which is strongly expressed at the endothelial tip of sprouting microvessels and plays a crucial role in the angiogenic response of the aortic rings, is also implicated in the reabsorption of the neovessels. Since the highest levels of MMP14 production in aortic culture are demonstrated when vessels have been completely reabsorbed (Aplin et al., 2009), which cell produces this enzyme in involuting aortic cultures? Morphologic analysis of aortic cultures
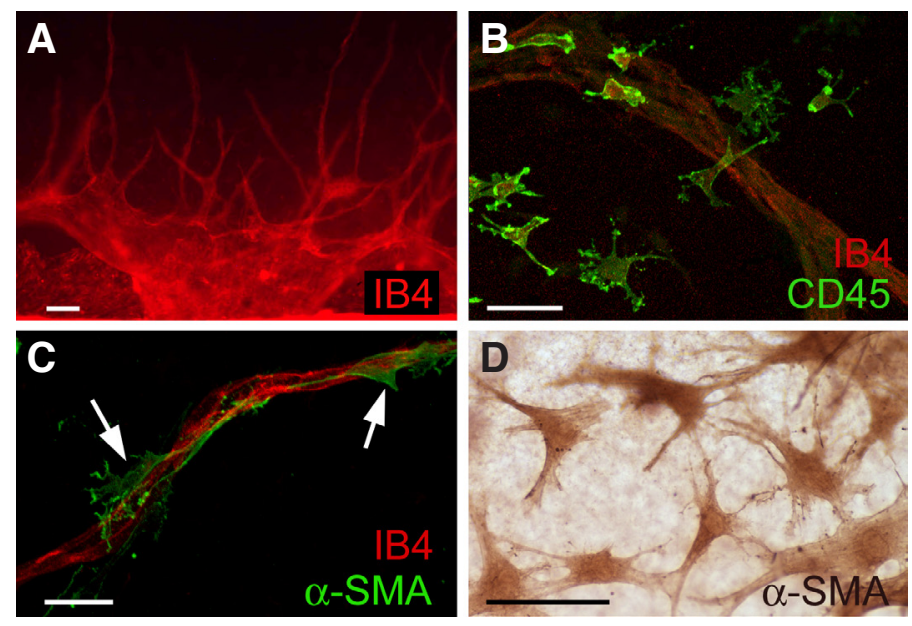

Fig. 4. Characterization of different cell types in aortic ring cultures by immunofluorescence (A-C) or immunoperoxidase (D). (A) Angiogenic outgrowths highlighted with the endothelial cell marker Griffonia Simplicifolia isolectin B4 (IB4). (B) CD45 positive macrophages are shown next to a vessel marked with IB4. (C) Aortic neovessel double stained for IB4 and the mural cell marker $\alpha$-SMA; Mural cells are marked with arrows. (D) Myofibroblasts of aortic outgrowths stained for $\alpha$-SMA. Scale bars, 100 microns. demonstrates that macrophages accumulate in areas of collagen lysis of regressing outgrowths in parallel with MMP14. RT-PCR and immunocytochemical analysis show that aortic macrophages are a rich source of MMPs including MMP14 produced during vascular regression (unpublished observations). Taken together, these findings suggest that macrophages switch from a proangiogenic to an angiolytic phenotype during vascular regression, and use MMPs including MMP14 to facilitate the reabsorption of the neovessels.

\section{Role of fibroblasts as promoters of angiogenesis}

Mesenchymal cells with morphologic features of fibroblasts are identified in the gel matrix around the aortic explants in 1-2-dayold cultures, prior to endothelial sprouting. As endothelial sprouts emerge from the aortic explants, elongate, branch, and eventually mature, fibroblasts migrate into the interstitial matrix between the vessel outgrowths and focally interact through cytoplasmic cell processes with the pericytes and the sprouting endothelium. All of the outgrowing fibroblasts stain for vimentin and many of them show features of myofibroblasts and express $\alpha$-smooth muscle actin ( $\alpha$-SMA; Fig. 4). Although the biologic significance in the angiogenic response of the early emergence of fibroblasts remains unclear, it is possible that these cells scout the extracellular matrix and serve as path-finders for the sprouting endothelium. There is also evidence that fibroblasts are involved in endothelial growth and survival mechanisms and play a central role in the contraction of the extracellular matrix (Majno et al., 1971). Fibroblasts isolated from rat tail tendons stimulate angiogenesis when co-embedded with aortic rings in the same collagen gel. The proangiogenic effect of fibroblasts is mediated by soluble factors since the angiogenic response of aortic rings in collagen gels can be stimulated with fibroblast conditioned medium (Villaschi and Nicosia, 1994). Endothelial cells isolated from aortic rings reorganize overnight into networks of branching microvessels when sandwiched between two layers of collagen. These endothelial tube networks, however, disintegrate in a few days if cultured in serum-free medium. Addition of fibroblasts to the collagen gel stabilize the microvessels and prevent their regression in the absence of serum supplements (Villaschi and Nicosia, 1994). Fibroblasts in these co-cultures develop myofibroblastic features and become closely associated with the microvascular networks, surrounding the endothelium with cytoplasmic processes. The proangiogenic activity of fibroblasts in co-culture with endothelial cells may be mediated by TIMP1 which promotes vessel survival in aortic ring cultures and, unlike the other TIMPs which are anti-angiogenic, stimulates the aortic angiogenic response (Liu et al., 2008). On the other hand the transformation of fibroblasts into contractile cells is probably mediated by endothelial derived endothelin-1 which promotes the expression in fibroblasts of $\alpha$-SMA (Villaschi and Nicosia, 1994). An additional factor potentially involved in the paracrine cross-talk between endothelial cells and fibroblasts is TGF $\beta 1$ which is produced by endothelial cells, activated in endothelial-mesenchymal cell co-cultures, and implicated in the induction of $\alpha$-SMA expression (Antonelli-Orlidge et al., 1989; Ding et al., 2004).

\section{Mechanisms of mural cell recruitment}

Periendothelial cells recruited by neovessels during aortic angiogenesis characteristically express markers of mural cell 
differentiation including $\alpha$-SMA and NG2 (Zhu and Nicosia, 2002; Howson et al., 2005). The unidirectional migration and proliferation of mural cells from the vessel roots toward the endothelial tip cells strongly implicate the existence of endothelial paracrine stimuli involved in the formation of the vessel wall. As predicted by studies with genetically modified mice (Hellstrom et al., 2001), PDGFB produced by sprouting endothelial cells is one of the endogenous promoters of mural cell recruitment in aortic cultures. Treatment with an anti-PDGFB antibody significantly decreases the number of mural cells in the aortic neovessels (Nicosia, 2009). Recruitment of mural cells can be enhanced by Ang1 which plays a critical role in the maturation and remodeling of neovessels during embryonal development (lurlaro et al., 2003). Ang1 promotes the assembly of the vessel wall by directly stimulating mural progenitor cells that express the Ang1 receptor Tie2 (lurlaro et al., 2003; Howson et al., 2005) or by indirectly stimulating mural cells through the induction in endothelial cells of chemotactic factors such as CCL2 (Aplin et al., 2010). Interestingly, mural cell recruitment is also stimulated by Ang2 (lurlaro et al., 2003), which was initially reported as an antagonist of Ang1 (Maisonpierre et al., 1997), but later recognized as a potential Tie2 activator whose agonist function was strongly influenced by contextual cues (Teichert-Kuliszewska et al., 2001). The migration and proliferation of mural cells is critically dependent on the p38 MAPK signaling pathway which can be activated by a number of angiogenic regulators including PDGFB and Ang1 (Yamaguchi et al., 2001; Zhu et al., 2002). Neutralization of this pathway with a 38 MAPK inhibitor or an adenoviral vector carrying a dominant negative form of $p 38$ suppresses mural cell recruitment without significantly affecting endothelial sprouting. Conversely transduction of aortic cultures with an adenovirus carrying MKK6, an upstream activator of p38 MAPK, potentiates the mural cell recruitment process (Zhu et al., 2003a). Mural cells are known to generate paracrine stimuli that stabilize neovessels and promote their survival. Our observation that vessels formed in aortic cultures eventually regress indicate that mural cells operating in an ex vivo environment have a reduced capacity to promote vascular survival. This may be due to the lack in aortic cultures of blood flow and mechanochemical signals that stimulate the vessel wall in the live animal.

\section{Stimulation of aortic angiogenesis by platelets}

Besides the lack of blood flow, one of the limitations of the aortic ring assay is the absence of cell types not represented in the aortic wall. This limitation can be overcome by supplementing the aortic cultures with exogenous cells of interest. For example the angiogenic response of the aortic rings can be stimulated by adding platelets to the cultures (Brill et al., 2004). Platelets induce vessel growth in a dose-dependent manner, and are maximally stimulatory at physiological densities. Asimilar effect can be obtained by treating aortic culture with platelet releasate. The angiogenic response of the aortic rings to platelets is reduced by treating the cultures with an inhibitor of VEGF receptor phosphorylation or an anti-bFGF mAb but not with an anti-PDGF B antibody. Maximal inhibition of angiogenesis is obtained by treating aortic ring-platelet co-cultures with a combination of VEGF, bFGF and PDGF inhibitors. The stimulatory effect of platelets on aortic angiogenesis can be potentiated by antibody-mediated blocking of platelet factor- 4 (PF-4), an anti-angiogenic factor present in the platelet releasate, or by activating platelets with thrombin (Brill et al., 2004).

\section{Paracrine interactions between cancer cells and aortic neovessels}

Angiogenesis in the aortic ring model can be stimulated by adding cancer cells to the aortic cultures. For example, aortic rings-containing plasma clots (or collagen gels) can be floated in the growth medium conditioned by planar cultures of sarcoma 180 cells (Nicosia and Ottinetti, 1990). Angiogenic factors produced by cancer cells diffuse into the gel matrix and stimulate the angiogenic response of the aortic explants.

Interactions between aortic neovessels and cancer cells can also be studied by co-embedding aortic rings and cancer cells in the same gel. Aggregates of rat bladder carcinoma cells embedded in fibrin clot without aortic rings grow slowly and by expansion. When aortic neovessels contact the tumor aggregates, carcinoma cells infiltrate the fibrin clot, migrating and proliferating in periendothelial location. Some vascular channels are disrupted and permeated by cords of invading cancer cells. Invading cancer cells are intimately associated with and attached to the subendothelial extracellular matrix (Nicosia et al., 1986; Nicosia et al., 1983). These observations demonstrate that neovessels formed ex vivo, i.e., in absence of active circulation, have the capacity to stimulate the spread of a carcinoma in plasma clot and modify its pattern of growth from expansile into highly invasive. One possible explanation for this phenomenon is that cancer cells use the extracellular matrix produced by endothelial cells and pericytes as an adhesive path for migration (Nicosia et al., 1986). It is also likely that cancer cells co-opt the neovessels in response to gradients of growth factors, cytokines and chemokines of endothelial/periendothelial cell origin (Rak et al., 1996). Finally, the angiogenic outgrowths create a network of extracellular matrix tunnels which may provide paths of least resistance for the invasion of cancer cells (Davis et al., 2007; Inai et al., 2004).

\section{Summary and Conclusion}

Studies with the aortic ring model have demonstrated that the angiogenic process can be reproduced and analyzed ex vivo by culturing vessel explants in three dimensional gels of extracellular matrix. Using this model we have discovered that the aortic wall has the capacity to produce all the necessary factors for the growth and maturation of a neovasculature. Formation of neovessels in this system is regulated by a complex interplay between resident immunocytes, endothelial cells, pericytes, and fibroblasts. Mechanical injury of the vessel wall triggers a self-limited cascade of gene activation that drives endothelial migration, proliferation, capillary tube formation, branching morphogenesis, and ultimately vascular regression. Although we have made significant progress in our understanding of the aortic ring model, our knowledge of the mechanisms that drive angiogenesis in this system remains incomplete. For example it is unknown how macrophages sense the injury of the dissection procedure and transduce this signal into proangiogenic stimuli. The molecular regulation of vascular branching morphogenesis and anastomosis formation has not been investigated. Particularly unclear are the mechanisms that regulate the vascular regression process and the production of angiolytic enzymes during the late stages of the angiogenic response. Ongoing 
studies aimed at characterizing different stages of the angiogenic cascade at a molecular and cellular level may provide new insights into how vessels form, mature, and are eventually reabsorbed in this model. By analyzing the angiogenic cascade in the aortic cultures we may ultimately identify novel molecular targets for the inhibition or stimulation of angiogenesis in pathologic conditions.

\section{Acknowledgments}

Supported by National Heart, Lung, and Blood Institute Grant HL52585 and Merit Review Grant from US Department of Veterans Affairs, Office of Research and Development, Biomedical Laboratory Research Program.

\section{References}

AMIN MA, VOLPERT OV, WOODS JM, KUMAR P, HARLOW LA, KOCH AE (2003). Migration inhibitory factor mediates angiogenesis via mitogen-activated protein kinase and phosphatidylinositol kinase. Circ Res 93: 321-329.

ANTONELLI-ORLIDGE A, SAUNDERS KB, SMITH SR, D'AMORE PA (1989). An activated form of transforming growth factor beta is produced by cocultures of endothelial cells and pericytes. Proc Natl Acad Sci USA 86: 4544-4548.

APLIN AC, FOGEL E, NICOSIA RF (2010). MCP-1 Promotes Mural Cell Recruitment During Angiogenesis in the Aortic Ring Model. Angiogenesis In Press: DOI: 10.1007/s10456-010-9179-8.

APLIN AC, FOGEL E, ZORZI P, NICOSIA RF (2008). The aortic ring model of angiogenesis. Methods Enzymol 443: 119-136.

APLIN AC, GELATI M, FOGEL E, CARNEVALE E, NICOSIA RF (2006). Angiopoietin-1 and vascular endothelial growth factor induce expression of inflammatory cytokines before angiogenesis. Physiol Genomics 27: 20-28.

APLINAC, ZHUWH, FOGELE, NICOSIARF (2009). Vascular regression and survival are differentially regulated by MT1-MMP and TIMPs in the aortic ring model of angiogenesis. Am J Physiol Cell Physiol 297: C471-C480.

AUERBACH R, LEWIS R, SHINNERS B, KUBAI L, AKHTAR N (2003). Angiogenesis assays: a critical overview. Clin Chem 49: 32-40.

BRILLA, ELINAV H, VARON D (2004). Differential role of platelet granular mediators in angiogenesis. Cardiovasc Res 63: 226-235.

CARMELIET P (2003). Angiogenesis in health and disease. Nat Med 9: 653-660.

DAVIS GE, KOHW, STRATMANAN (2007). Mechanisms controlling human endothelial lumen formation and tube assembly in three-dimensional extracellular matrices. Birth Defects Res C Embryo Today 81: 270-285.

DIGLIO CA, GRAMMAS P, GIACOMELLI F, WIENER J (1989). Angiogenesis in rat aorta ring explant cultures. Lab Invest 60: 523-531.

DING R, DARLAND DC, PARMACEK MS, D'AMORE PA (2004). Endothelial-mesenchymal interactions in vitro reveal molecular mechanisms of smooth muscle/ pericyte differentiation. Stem Cells Dev 13: 509-520.

EMANUELS, GRUNINGERRH, FUENTES-PESQUERAA, CONNOLLYPJ, SEAMON JA, HAZEL S, TOMINOVICH R, HOLLISTER B, NAPIER C, D'ANDREA MR, REUMAN M, BIGNAN G, TUMAN R, JOHNSON D, MOFFATT D, BATCHELOR M, FOLEYA, O'CONNELLJ, ALLEN R, PERRY M, JOLLIFFE L, MIDDLETON SA (2004). A vascular endothelial growth factor receptor-2 kinase inhibitor potentiates the activity of the conventional chemotherapeutic agents paclitaxel and doxorubicin in tumor xenograft models. Mol Pharmacol 66: 635-647.

GELATI M, APLIN AC, FOGEL E, SMITH KD, NICOSIA RF (2008). The angiogenic response of the aorta to injury and inflammatory cytokines requires macrophages. $J$ Immunol 181: 5711-5719.

HELLSTROM M, GERHARDT $\mathrm{H}$, KALEN M, LI X, ERIKSSON U, WOLBURG $\mathrm{H}$, BETSHOLTZ C (2001). Lack of pericytes leads to endothelial hyperplasia and abnormal vascular morphogenesis. J Cell Biol 153: 543-553.

HOWSON KM, APLIN AC, GELATI M, ALESSANDRI G, PARATI EA, NICOSIA RF (2005). The postnatal rat aorta contains pericyte progenitor cells that form spheroidal colonies in suspension culture. Am J Physiol Cell Physiol 289: C1396-C1407.

HUH SJ, CHUNG CY, SHARMA A, ROBERTSON GP (2010). Macrophage inhibitory cytokine-1 regulates melanoma vascular development. Am J Pathol 176: 2948-2957.

INAI T, MANCUSO M, HASHIZUME H, BAFFERT F, HASKELL A, BALUK P, HU-
LOWE DD, SHALINSKY DR, THURSTON G, YANCOPOULOS GD, MCDONALD DM (2004). Inhibition of vascular endothelial growth factor (VEGF) signaling in cancer causes loss of endothelial fenestrations, regression of tumor vessels, and appearance of basement membrane ghosts. Am J Pathol 165: 35-52.

IURLARO M, SCATENA M, ZHU WH, FOGEL E, WIETING SL, NICOSIA RF (2003). Rat aorta-derived mural precursor cells express the Tie2 receptor and respond directly to stimulation by angiopoietins. J Cell Sci 116: 3635-3643.

LEIBOVICH SJ, POLVERINI PJ, SHEPARD HM, WISEMAN DM, SHIVELYV, NUSEIR $N$ (1987). Macrophage-induced angiogenesis is mediated by tumour necrosis factor-alpha. Nature 329: 630-632.

LIU H, CHENB, LILLYB (2008). Fibroblasts potentiate blood vessel formation partially through secreted factor TIMP-1. Angiogenesis 11: 223-234.

MAISONPIERRE PC, SURI C, JONES PF, BARTUNKOVA S, WIEGAND SJ, RADZIEJEWSKI C, COMPTON D, MCCLAIN J, ALDRICH TH, PAPADOPOULOS N, DALY TJ, DAVIS S, SATO TN, YANCOPOULOS GD (1997). Angiopoietin-2, a natural antagonist for Tie2 that disrupts in vivo angiogenesis. Science 277: 55-60.

MAJNO G, GABBIANI G, HIRSCHELBJ, RYAN GB, STATKOV PR (1971). Contraction of granulation tissue in vitro: similarity to smooth muscle. Science 173: 548-550.

MASSON V, DEVY L, GRIGNET-DEBRUS C, BERNT S, BAJOU K, BLACHER S, ROLAND G, CHANG Y, FONG T, CARMELIET P, FOIDART J, NOEL A (2002). Mouse Aortic Ring Assay: A New Approach of the Molecular Genetics of Angiogenesis. Biol Proced Online 4: 24-31.

MORI M, SADAHIRA Y, KAWASAKI S, HAYASHI T, NOTOHARA K, AWAI M (1988). Capillary growth from reversed rat aortic segments cultured in collagen gel. Acta Pathol Jpn 38: 1503-1512.

NICOSIA R (2009). The Aortic Ring Model of Angiogenesis: A Quarter Century of Search and Discovery. J Cell Mol Med 13: 4113-4136.

NICOSIARF, BONANNOE, VILLASCHIS (1992). Large-vessel endothelium switches to a microvascular phenotype during angiogenesis in collagen gel culture of rat aorta. Atherosclerosis 95: 191-199.

NICOSIA RF, LIN YJ, HAZELTON D, QIAN X (1997). Endogenous regulation of angiogenesis in the rat aorta model. Role of vascular endothelial growth factor. Am J Pathol 151: 1379-1386.

NICOSIA RF, NICOSIA SV, SMITH M (1994a). Vascular endothelial growth factor, platelet-derived growth factor, and insulin-like growth factor-1 promote rat aortic angiogenesis in vitro. Am J Pathol 145: 1023-1029.

NICOSIARF, OTTINETTIA (1990). Growth of microvessels in serum-free matrix culture of rat aorta. A quantitative assay of angiogenesis in vitro. Lab Invest 63: 115-122.

NICOSIARF, TCHAOR, LEIGHTON J (1983). Angiogenesis-dependent tumor spread in reinforced fibrin clot culture. Cancer Res 43: 2159-2166.

NICOSIA RF, TCHAO R, LEIGHTON J (1986). Interactions between newly formed endothelial channels and carcinoma cells in plasma clot culture. Clin Exp Metastasis 4: 91-104.

NICOSIA RF, VILLASCHI S (1995). Rat aortic smooth muscle cells become pericytes during angiogenesis in vitro. Lab Invest 73: 658-666.

NICOSIA RF, VILLASCHI S, SMITH M (1994b). Isolation and characterization of vasoformative endothelial cells from the rat aorta. In vitro Cell Dev Biol Anim 30A: 394-399.

NICOSIA RF, ZHU WH, FOGEL E, HOWSON KM, APLIN AC (2005). A new ex vivo model to study venous angiogenesis and arterio-venous anastomosis formation. $J$ Vasc Res 42: 111-119.

NILSSON MB, LANGLEY RR, FIDLER IJ (2005). Interleukin-6, secreted by human ovarian carcinoma cells, is a potent proangiogenic cytokine. Cancer Res 65 10794-10800.

PATAN S (2004). Vasculogenesis and angiogenesis. Cancer Treat Res 117: 3-32.

RAK J, FILMUS J, KERBEL RS (1996). Reciprocal paracrine interactions between tumour cells and endothelial cells: the 'angiogenesis progression' hypothesis. Eur J Cancer 32A: 2438-2450.

RIBATTI D, VACCA A (1999). Models for studying angiogenesis in vivo. Int J Biol Markers 14: 207-213.

SALCEDO R, WASSERMAN K, YOUNG HA, GRIMM MC, HOWARD OM, ANVER MR, KLEINMAN HK, MURPHY WJ, OPPENHEIM JJ (1999). Vascular endothelia growth factor and basic fibroblast growth factor induce expression of CXCR4 on human endothelial cells: In vivo neovascularization induced by stromal-derived factor-1alpha. Am J Pathol 154: 1125-1135. 
STRIETER RM, BELPERIO JA, BURDICK MD, SHARMAS, DUBINETT SM, KEANE MP (2004). CXC chemokines: angiogenesis, immunoangiostasis, and metastases in lung cancer. Ann N Y Acad Sci 1028: 351-360.

TEICHERT-KULISZEWSKA K, MAISONPIERRE PC, JONES N, CAMPBELL AI, MASTER Z, BENDECK MP, ALITALO K, DUMONT DJ, YANCOPOULOS GD, STEWART DJ (2001). Biological action of angiopoietin-2 in a fibrin matrix model of angiogenesis is associated with activation of Tie2. Cardiovasc Res 49: 659-670.

UCUZIAN AA, GREISLER HP (2007). In vitro models of angiogenesis. World J Surg 31: 654-663.

VILLASCHI S, NICOSIA RF (1993). Angiogenic role of endogenous basic fibroblast growth factor released by rat aorta after injury. Am J Pathol 143: 181-190.

VILLASCHI S, NICOSIA RF (1994). Paracrine interactions between fibroblasts and endothelial cells in a serum-free coculture model. Modulation of angiogenesis and collagen gel contraction. Lab Invest 71: 291-299.

YAMAGUCHI H, IGARASHI M, HIRATA A, TSUCHIYA H, SUSA S, TOMINAGA M DAIMON M, KATO T (2001). Characterization of platelet-derived growth factor- induced p38 mitogen-activated protein kinase activation in vascular smooth muscle cells. Eur J Clin Invest 31: 672-680.

ZHU WH, GUO X, VILLASCHI S, FRANCESCO NR (2000). Regulation of vascular growth and regression by matrix metalloproteinases in the rat aorta model of angiogenesis. Lab Invest 80: 545-555.

ZHU WH, HAN J, NICOSIA RF (2003a). Requisite role of p38 MAPK in mural cell recruitment during angiogenesis in the rat aorta model. J Vasc Res 40: 140-148.

ZHU WH, IURLARO M, MACINTYREA, FOGELE, NICOSIARF (2003b). The mouse aorta model: influence of genetic background and aging on bFGF- and VEGFinduced angiogenic sprouting. Angiogenesis 6: 193-199.

ZHU WH, MACINTYRE A, NICOSIA RF (2002). Regulation of angiogenesis by vascular endothelial growth factor and angiopoietin-1 in the rat aorta model: distinct temporal patterns of intracellular signaling correlate with induction of angiogenic sprouting. Am J Pathol 161: 823-830.

ZHU WH, NICOSIA RF (2002). The thin prep rat aortic ring assay: a modified method for the characterization of angiogenesis in whole mounts. Angiogenesis 5: 81-86. 


\section{Further Related Reading, published previously in the Int. J. Dev. Biol.}

Insulin-like growth factor-2 regulates early neural and cardiovascular system development in zebrafish embryos Lori Hartnett, Catherine Glynn, Catherine M. Nolan, Maura Grealy and Lucy Byrnes Int. J. Dev. Biol. (2010) 54: 573-583

The seminal work of Werner Risau in the study of the development of the vascular system Domenico Ribatti

Int. J. Dev. Biol. (2010) 54: 567-572

Estrogen regulation of placental angiogenesis and fetal ovarian development during primate pregnancy Eugene D. Albrecht and Gerald J. Pepe Int. J. Dev. Biol. (2010) 54: 397-407

Uteroplacental vascular development and placental function: an update Lawrence P. Reynolds, Pawel P. Borowicz, Joel S. Caton, Kimberly A. Vonnahme, Justin S. Luther, David S. Buchanan, Shireen A. Hafez, Anna T. Grazul-Bilska and Dale A. Redmer

Int. J. Dev. Biol. (2010) 54: 355-365

Critical growth factors and signalling pathways controlling human trophoblast invasion Martin Knöfler

Int. J. Dev. Biol. (2010) 54: 269-280

Over-expression of thymosin beta4 promotes abnormal tooth development and stimulation of hair growth Hee-Jae Cha, Deborah Philp, Soo-Hyun Lee, Hye-Sung Moon, Hynda K. Kleinman and Takashi Nakamura Int. J. Dev. Biol. (2010) 54: 135-140

The contribution of Roberto Montesano to the study of interactions between epithelial sheets and the surrounding extracellular matrix Domenico Ribatti

Int. J. Dev. Biol. (2010) 54: 1-6

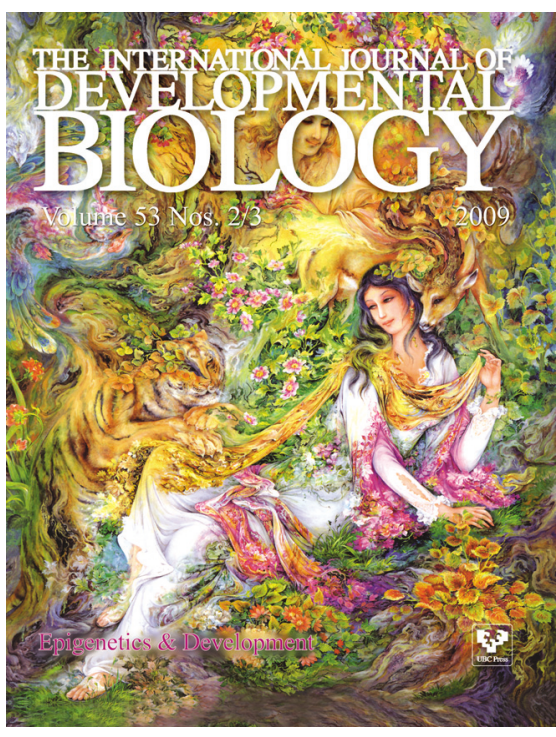

5 yr ISI Impact Factor $(2009)=3.253$

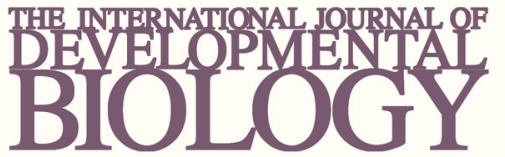

Volume 54 Nos. 6/7

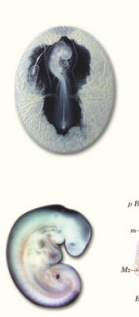

Developmental Hematopoiesis
Special Issue
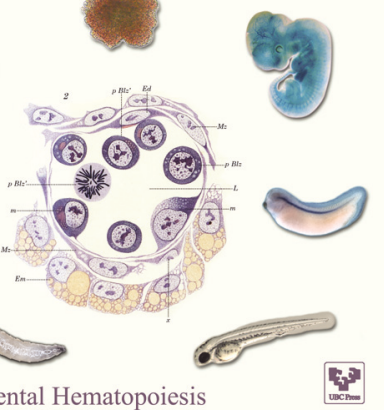

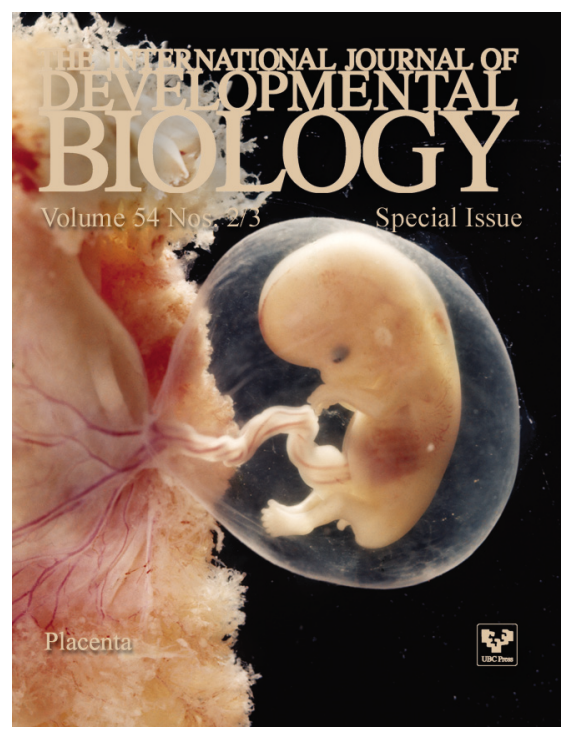

